Full Length Article

\title{
Light touch compensates peripheral somatosensory degradation in postural control of older adults
}

\author{
Ana M.F. Barela ${ }^{\mathrm{a}, *}$, Sarah Caporicci ${ }^{\mathrm{a}}$, Paulo Barbosa de Freitas ${ }^{\mathrm{a}}$, John J. Jeka ${ }^{\mathrm{c}}$, \\ José A. Barela ${ }^{\mathrm{a}, \mathrm{b}}$

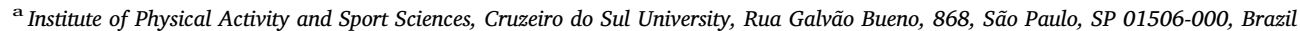 \\ ${ }^{\mathrm{b}}$ Department of Physical Education, São Paulo State University, Av. 24-A, 1515, Rio Claro, SP 13506-900, Brazil \\ ${ }^{\mathrm{c}}$ Department of Kinesiology \& Applied Physiology, University of Delaware, $201 T$ Health Science Complex, Newark, DE, United States
}

\section{A R T I C L E I N F O}

\section{Keywords:}

Body sway

Light touch fingertip

Passive joint movement

Proprioception

\begin{abstract}
A B S T R A C T
The present study aimed to investigate the sensitivity of detecting lower limb passive motion and use of additional sensory information from fingertip light touch for the postural control of older adults in comparison with young adults. A total of 11 older and 11 young adults (aged $68.1 \pm 5.2$ and $24.2 \pm 2.2$ years, respectively) underwent two tasks. We evaluated their sensitivity to passive ankle joint movement while seated in the first task. Participants then stood quietly on a force plate in a semi-tandem stance, for $30 \mathrm{~s}$ under two fingertip contact force conditions (no touch and light touch limited to $1 \mathrm{~N}$ ). The results showed that the threshold of passive ankle displacement and body sway is higher in older adults than in young adults. The body sway reduced for both older and young adults with the addition of light touch at the fingertips. The maximum cross-correlation coefficient and time lags between body sway and fingertip light touch center of pressure was similar between both groups, suggesting that older adults used light touch to reduce body sway, similar to young adults. A higher threshold in detecting passive ankle joint movement may contribute to the increased body sway observed in older adults. These deficits may be compensated by additional sensory cues that would provide enhanced information used to control the upright stance.
\end{abstract}

\section{Introduction}

Sensory feedback about the relationship established between adjacent body segments and the relationship between the wholebody configuration and the surrounding environment is crucial for maintaining upright stance. To provide useful information about body dynamics in space, sensory stimuli received from different sources (e.g., visual, vestibular, and somatosensory systems) (Nashner, 1981) need to be integrated in the central nervous system (CNS). Based on this information, appropriate muscle activation is produced to achieve or maintain a desirable body posture. Therefore, postural control requires appropriate sensory integration and an intricate relationship between the sensory and motor systems (Horak \& Macpherson, 1996).

The findings of several previous studies indicate that older adults present poorer postural control performance (i.e., larger body sway) than young adults (Lord \& Ward, 1994; Maki \& McIlroy, 1996; Sturnieks, St George, \& Lord, 2008; Woollacott \& ShumwayCook, 1990). Despite other possible factors, poorer postural control performance is associated with an impaired ability to obtain accurate afferent cues from the body periphery (Blaszczyk, Lowe, \& Hansen, 1994; Collins, De Luca, Burrows, \& Lipsitz, 1995; Lord \&

\footnotetext{
* Corresponding author at: Rua Galvão Bueno, 868, 130 andar, Bloco B, São Paulo, SP 01506-000, Brazil.

E-mail addresses: ana.barela@cruzeirodosul.edu.br (A.M.F. Barela), paulo.defreitas@cruzeirodosul.edu.br (P.B. de Freitas), jjeka@udel.edu (J.J. Jeka), jose.barela@cruzeirodosul.edu.br (J.A. Barela).
} 
Menz, 2000; Prioli, Cardozo, de Freitas Junior, \& Barela, 2006; Prioli, Freitas Junior, \& Barela, 2005). For instance, Toledo and Barela (2010, 2014) recently demonstrated that less accurate detection of passive motion around the ankle joint was associated with larger sway in older adults during the upright stance. Assuming that in an upright stance, the human body behaves as a single-segment inverted pendulum (Winter, Prince, Frank, Powell, \& Zabjek, 1996), information around the ankle is critical to detect any whole-body deviation from its desirable position. Therefore, if older adults need larger displacement to detect passive motion in their ankles, their greater sway could be directly related to less accurate lower limb proprioceptive cues (Toledo \& Barela, 2010).

In a situation in which available sensory cues are not accurate, creating less precise estimation of body dynamics, an alternative strategy would be to enhance information by providing alternative sensory cue sources. For instance, several studies demonstrated that information from fingertip contact to a rigid surface provides sensory cues that can be used as an additional source of somatosensory information to attenuate body sway in upright stance in newly walking infants (Barela, Jeka, \& Clark, 1999; Metcalfe et al., 2005), children (Barela, Jeka, \& Clark, 2003), young adults (Holden, Ventura, \& Lackner, 1994; Jeka \& Lackner, 1994, 1995), and in individuals with vestibular dysfunction (Lackner et al., 1999), peripheral neuropathy (Dickstein, Shupert, \& Horak, 2001), anterior cruciate ligament injury (Bonfim, Grossi, Paccola, \& Barela, 2008), and stroke (Cunha, Alouche, Araujo, \& Freitas, 2012). The premise of this approach is that fingertip contact to a rigid surface provides information about arm position and body sway, leading to anticipatory muscle activity to revert and/or maintain a more stable upright stance, consequently, reducing the body sway magnitude (Jeka \& Lackner, 1994, 1995; Jeka, Schoner, Dijkstra, Ribeiro, \& Lackner, 1997).

A few studies investigated the effects of light touch on body sway of older adults (Baccini et al., 2007; Reginella, Redfern, \& Furman, 1999; Tremblay, Mireault, Dessureault, Manning, \& Sveistrup, 2004). Although they employed different methodology, the studies revealed that older adults were able to reduce body sway by touching a stationary surface. Baccini and colleagues (2007) showed that by lightly contacting (applying forces up to $1 \mathrm{~N}$ ) a rigid surface, older adults reduced their body sway compared to a noncontact condition; the effect of light touch was even larger compared to that observed in young adults. Similarly, Tremblay and colleagues (2004) also observed a reduction in body sway in older adults, although they did not control the amount of applied force on the touching surface. The use of fingertip cues from contacting a surface to reduce body sway magnitude in older adults was even observed when cues from the lower limbs were distorted (Reginella et al., 1999). Despite the important and promising findings from these studies (Baccini et al., 2007; Reginella et al., 1999; Tremblay et al., 2004), however, there are many questions related to how older adults use light touch on a surface to reduce postural sway, which remain to be examined. First, the relationship between changes in the applied fingertip forces and body sway has not been assessed in older adults. In young adults, changes in applied forces preceded (approximately $300 \mathrm{~ms}$ ) the corresponding body sway, indicating that changes in applied forces at the fingertip are used to anticipate changes in body sway (Jeka \& Lackner, 1995). Second, the aforementioned studies that investigated the effect of light touching in older adults (Reginella et al., 1999; Tremblay et al., 2004) did not limit the applied force to 1 N. Thus, it is uncertain whether the light touch provided a sensory or mechanical effect. Therefore, although contacting a surface to reduce the magnitude of body sway has been established in older adults, the mechanism underlying the use of such contact for body stabilization still requires further investigation.

Despite the possible questions related to the use of enhanced somatosensory cues from the fingertip contact by older adults, Baccini and colleagues (2007) suggested that tactile cues obtained from contacting a rigid surface might counterbalance detrimental deterioration of sensory information from the lower extremities, which can compromise postural performance in older adults. Results from Tremblay and colleagues (2004) corroborated with this hypothesis, since they demonstrated that for older adults fingertip contact to a stationary surface is useful for controlling body sway, even when proprioceptive cues from the lower limbs were distorted. The use of additional sensory cues, such as the ones provided by lightly touching a rigid surface to improve upright stance performance, is important because previous findings indicate that body sway in older adults is influenced by less accurate motion detection around the ankle (Lord, Clark, \& Webster, 1991; Lord \& Menz, 2000; Toledo \& Barela, 2010; Winter et al., 1996). Thus, older adults might enhance sensory information by additional cues coming from lightly touching a rigid surface to compensate impairments in their lower limb proprioception acuity. Assuming that light touch and motion detection around the ankle joint do not share the same functioning mechanisms (i.e., receptors responding to different stimuli and having different thresholds), the CNS might take advantage of using cues coming from both sources, i.e., light touch and motion around the ankle joint, to improve postural control performance. However, if cues from the motion around the ankle joint are degraded with age, the CNS would obtain less accurate information about body dynamics, e.g. sway position and velocity. Thus, contacting a surface would be sufficient to compensate for such sensory degradation to maintain a performance level similar to young adults.

Despite evidence that older adults can use light touch to reduce body sway (Baccini et al., 2007; Tremblay et al., 2004), and its possible behavioral role in compensating for deteriorated proprioceptive cues from lower limbs, this direct relationship has not been examined yet. Therefore, the main aim of the present study was to examine whether possibly less accurate detection of passive motion of ankle joint would account for larger sway of older adults. In addition, this study also examined the use of light touch by older adults to determine if it might be sufficient to overcome possibly less accurate cues coming from the lower limbs. We hypothesized that older adults would be less sensitive to passive motion detection in the lower limb joint and in postural control when compared to young adults. However, such deterioration of lower limb sensitivity in older adults would be compensated by sensory cues from the fingertip contacting a rigid surface to reduce postural sway. 


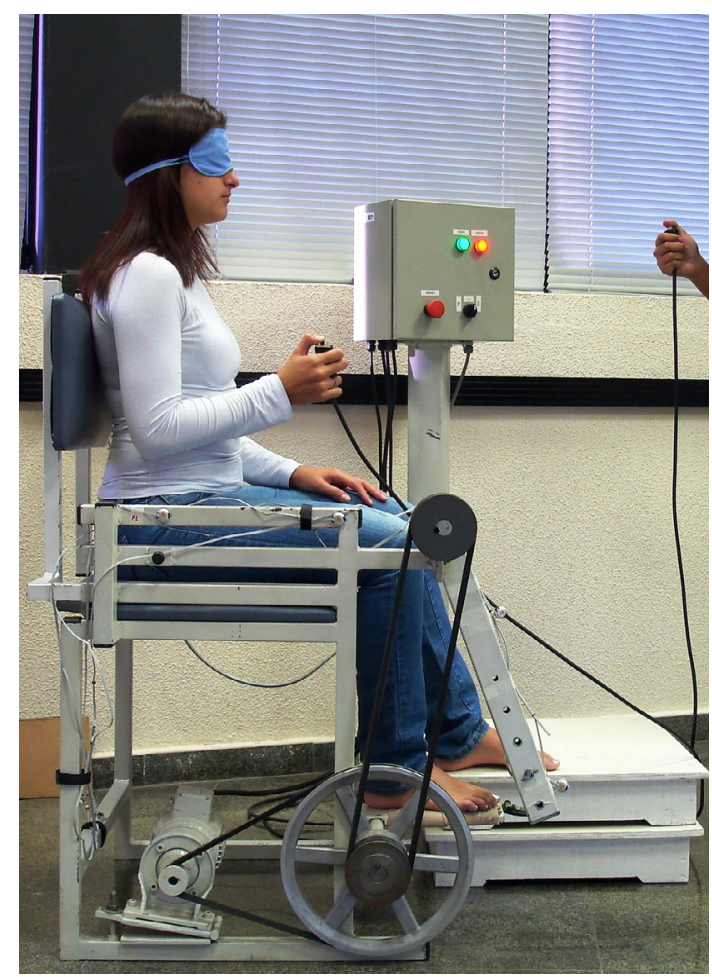

Fig. 1. Representative illustration of a person seated on the "passive motion detector chair" with her right foot placed on the foot stand in the neutral position and holding the cylindrical implement. She provided written informed consent to publish this image.

\section{Materials and methods}

\subsection{Participants}

A total of 11 older ( 7 women, 4 men) and 11 young ( 8 women, 3 men) adults, with a mean age ( \pm SD) of $68.1 \pm 5.2$ and $24.2 \pm 2.2$ years, respectively, participated in the present study. All participants were healthy, with no known musculoskeletal injuries or neurological disorders, which could impair their ability to maintain balance. They had normal or corrected-to-normal vision and were self-reported right foot dominant. The Institutional Review Ethics Board of the Cruzeiro do Sul University approved the procedures of this study. All participants provided informed consent before the experimental session.

\subsection{Apparatus}

Customized equipment based on the continuous passive motion (Stryker Leg Exerciser) was used to assess passive ankle joint motion sensitivity (Fig. 1). This equipment ("passive motion detector chair") consisted of a chair with a right foot stand, which could be rotated upward or downward, causing ankle dorsiflexion or ankle plantar flexion, respectively, controlled by a servo-motor with angular velocity of $0.5^{\circ} \mathrm{s}$. Three infrared emitters of a motion capture system (OPTOTRAK Certus, NDI, Inc.) were placed in both extremities of the foot stand and in the chair. Linear kinematic data were used to calculate the angular displacement of the foot stand. Smaller angular displacement indicated better sensitivity to joint passive motion.

To assess the postural sway and the force exerted by the fingertip on the rigid surface, a force platform (Kistler, Model 9286A) and a multi-axis force/torque (F/T) transducer (Nano 17 Titanium, ATI, Inc.) were used. The F/T transducer was mounted on a tripod, which could be adjusted in height and position to allow a comfortable arm position as participants touched the contact surface with their right index fingertip. The top surface of the F/T transducer in which the participants placed the tip of their index finger was covered with sandpaper (200 grit) to provide a high coefficient of friction between skin and the touch contact surface, assuring a high magnitude of cutaneous stimuli.

\subsection{Tasks and procedures}

To assess ankle joint passive motion sensitivity, participants were asked to remain seated in the passive motion detector chair with the right foot placed on the foot stand, as the initial angle between shank and foot was $90^{\circ}$ (neutral position) (Fig. 1). They were also asked to hold a small cylindrical implement (plastic canister) with a push switch on the top using their right hand and instructed to 
press the switch, as soon as they perceived that their right foot was passively moved, in order to interrupt the movement, which was triggered by the experimenter. During data acquisition, participants were blindfold to guarantee the absence of visual information. Six balanced and randomized trials of dorsiflexion and plantar flexion movements were acquired at a frequency of $100 \mathrm{~Hz}$. An experimenter initiated each trial with a verbal "go" signal.

To assess postural control, participants were asked to maintain an upright semi-tandem stance (hallux of right foot touching the medial border of calcaneus of the left foot) on the force platform in two visual conditions, i.e., vision and no vision (although we only analyzed data from the "no vision" condition in the present study), and two fingertip contact conditions as follows: no contact, in which participants' arms hung passively beside their bodies; and light contact, in which participants were asked to place the tip of their right index finger on the touch contact surface and apply up to $1 \mathrm{~N}$ of vertical force on this surface. During the trials with vision, participants looked straight ahead towards a target (with a 3-cm diameter) placed $1 \mathrm{~m}$ away at eye level. During the no vision trials, they remained with their eyes closed, looking ahead as in the vision condition (with eyes open). Foot position was traced with a marker on top of the force platform to ensure identical foot positioning during all trials. The magnitude of vertical force applied by the fingertip on the contact surface was limited to $1 \mathrm{~N}$ and was controlled in real-time by an experimenter. If the vertical force exceeded $1 \mathrm{~N}$, a computer-generated auditory "beep" would indicate that the applied force level should be reduced. Considering that fingertip tactile sensitivity could also be degraded in older adults, we tested tactile sensitivity by using Semmes-Weinstein monofilaments.

The four conditions were distributed across into three blocks of randomized trials with 1 min of rest between each block. Three 30-s trials were recorded for each condition, with a total of 12 trials. Data were collected at a frequency of $100 \mathrm{~Hz}$, using a personal computer equipped with a data acquisition board (NI-BNC2090, National Instruments, Inc.) and a customized Labview program (Labview 2011, National Instruments, Inc, Austin, TX, USA).

\subsection{Data analysis}

Ankle joint passive motion sensitivity was calculated using custom software written in MATLAB (The MathWorks, Inc). Data were filtered using a fourth-order zero lag low-pass Butterworth digital filter, with a cut-off frequency of $6 \mathrm{~Hz}$. Joint passive motion sensitivity corresponded to the difference between the initial (neutral) and final (when chair motion was interrupted by the participant) angular position.

The first and last $2.5 \mathrm{~s}$ periods of data collection were excluded from analysis to minimize anticipation effects associated with the beginning and end of each trial, leaving a total of data collected over $25 \mathrm{~s}$ for each trial, from which the center of pressure (CP) from the force platform was obtained. Applied forces to the F/T transducer were also used to calculate the CP of fingertip contact force. Individual applied forces (horizontal, vertical) and CPs (force plate and F/T transducer) were filtered using a fourth-order zero lag low-pass Butterworth digital filter, with a cut-off frequency of $10 \mathrm{~Hz}$. The following measurements were also calculated using a customized MATLAB (The MathWorks, Inc.) routine as follows: 1) CP mean sway amplitude (MSA) in both anterior-posterior (AP) and medial-lateral (ML) directions, calculated by subtracting the average position of CP from each data point within a trial and calculating the standard deviation of this signal; 2) mean horizontal and vertical absolute forces applied by the fingertip; 3) maximum cross-correlation coefficient $\left(r_{\max }\right)$; and 4) the time lag between $\mathrm{CP}$ of the feet and $\mathrm{CP}$ of the fingertip. Negative time lags indicated that the changes in the $\mathrm{CP}$ of the feet preceded changes in the $\mathrm{CP}$ of the fingertip; positive time lags indicated the opposite, i.e., the $\mathrm{CP}$ of the feet followed the $\mathrm{CP}$ of the fingertip. Since correlations do not have a normal distribution, the data were first transformed to the Fischer's z values, prior to statistical analysis.

\subsection{Statistical analyses}

Data from individual trials were averaged within each condition (ankle joint displacement and postural control) for each participant. For the joint passive motion sensitivity, an analysis of variance (ANOVA) was employed, using group as a factor (young and older adults) and joint displacement as a dependent variable.

For postural control, a multivariate analysis (MANOVA) was employed, using group and fingertip contact (no touch, light touch) as factors, with repeated measures for the last factor; MSA-AP and MSA-ML were dependent variables. Two other MANOVAs were employed, using group as a factor, and the mean applied forces on the touch device in the three directions (vertical, AP and ML) as dependent variables, for the first MANOVA, and the $r_{\max }$ between $\mathrm{CP}$ of the feed and $\mathrm{CP}$ of the finger and its respective time lag in the light touch condition, for the second MANOVA. Fingertip tactile sensitivity was compared between groups using the Mann-Whitney nonparametric test.

Pearson correlation tests were employed to examine the relationship between joint passive motion sensitivity and body sway in the two experimental conditions (i.e., no touch and light touch). In addition, we had run a multivariate analysis of covariance (MANCOVA) with the same factors and dependent variables employed for the postural control, but having the ankle joint passive motion as a covariate. This last analysis was used to test whether the ankle joint sensitivity assessed by the ankle joint displacement would account for the difference in the postural sway magnitude observed between groups.

When applicable, univariate analyses and Tukey's HSD tests were employed. All the assumptions for the employed analyses were fulfilled and an alpha level of 0.05 was set for all statistical tests, which were performed using SPSS software. 


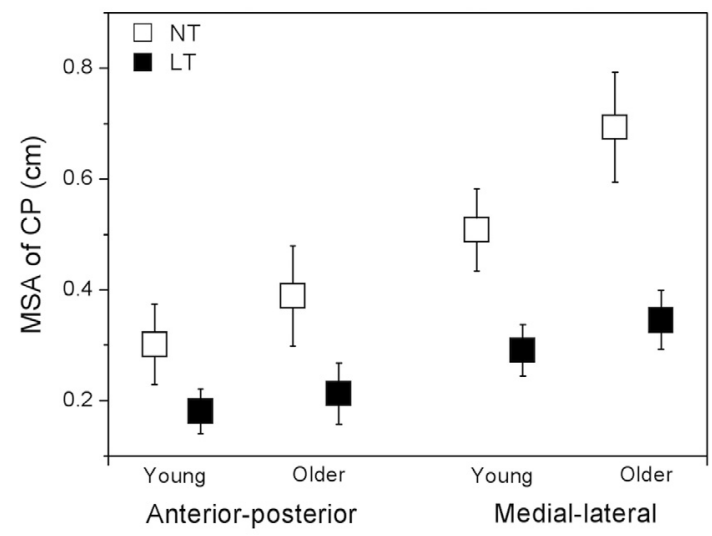

Fig. 2. Mean ( \pm SD) of the magnitudes of mean sway amplitude (MSA) of the center of pressure (CP) of young and older adults, during the anteriorposterior and medial-lateral directions during no touch (NT, open squares) and light touch (LT, filled squares) conditions.

\section{Results}

\subsection{Ankle joint passive motion sensitivity}

ANOVA revealed a group effect for joint passive motion $\left(\mathrm{F}_{1,20}=15.20, \mathrm{p}=0.001\right.$ partial $\left.\eta^{2}=0.432\right)$. Older adults presented higher passive joint displacement $(\mathrm{M}=1.43, \mathrm{SD}=0.65)$ than young adults $(\mathrm{M}=0.62, \mathrm{SD}=0.25)$.

\subsection{Postural control}

Fig. 2 shows the mean MSA (AP and ML) of CP values for young and older adults during the experimental conditions. MANOVA revealed a main effect of group (Wilks' Lambda $=0.37, \mathrm{~F}_{2,19}=15.92, \mathrm{p}<0.001$, partial $\eta^{2}=0.626$ ), touch (Wilks' Lambda $=0.10$, $\mathrm{F}_{2,19}=89.16, \mathrm{p}<0.001$, partial $\eta^{2}=0.904$ ), and touch by group interaction (Wilks' Lambda $=0.64, \mathrm{~F}_{2,19}=5.28, \mathrm{p}=0.015$, partial $\eta^{2}=0.357$ ). Univariate analysis revealed group (MSA-AP: $F_{1,20}=5.60, p=0.028$, partial $\eta^{2}=0.219$; MSA-ML: $\mathrm{F}_{1,20}=29.12, \mathrm{p}<0.001$, partial $\eta^{2}=0.593$ ), touch (MSA-AP: $\mathrm{F}_{1,20}=111.55, \mathrm{p}<0.001$, partial $\eta^{2}=0.848$; MSA-ML: $\mathrm{F}_{1,20}=186.70, \mathrm{p}<0.001$, partial $\eta^{2}=0.903$ ), and touch by group interaction (MSA-ML: $\mathrm{F}_{1,20}=10.01, \mathrm{p}=0.005, \mathrm{partial}$ $\eta^{2}=0.333$ ) effects. Overall, older adults presented greater MSA than young adults. Further, the MSA was larger with no touch than with light touch in both directions (AP and ML). Tukey post hoc analyses indicated that with no touch, older adults swayed more in the ML direction, compared to young adults ( $\mathrm{p}<0.001$ ); with light touch, however, the sway was similar for both groups (p > 0.05) (Fig. 2).

\subsection{Applied forces and postural control}

Fig. 3 presents mean absolute applied forces for both age groups during the conditions with fingertip contact. MANOVA revealed a group effect (Wilks' Lambda $=0.46, \mathrm{~F}_{3,18}=6.90, \mathrm{p}=0.003$, partial $\eta^{2}=0.535$ ). Univariate analysis revealed group effects for the vertical $\left(\mathrm{F}_{1,20}=19.02, \mathrm{p}<0.001\right.$, partial $\left.\eta^{2}=0.487\right)$, AP $\left(\mathrm{F}_{1,20}=15.01, \mathrm{p}=0.001\right.$, partial $\left.\eta^{2}=0.429\right)$, and $\mathrm{ML}\left(\mathrm{F}_{1,20}=9.98\right.$,

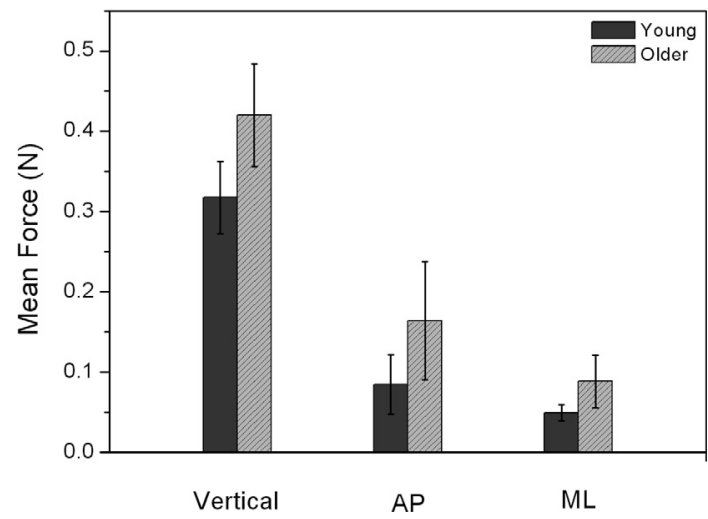

Fig. 3. Mean $( \pm S D)$ of the mean absolute applied forces in the vertical, anterior-posterior (AP), and medial-lateral (ML) directions of young and older adults during the light touch conditions. 


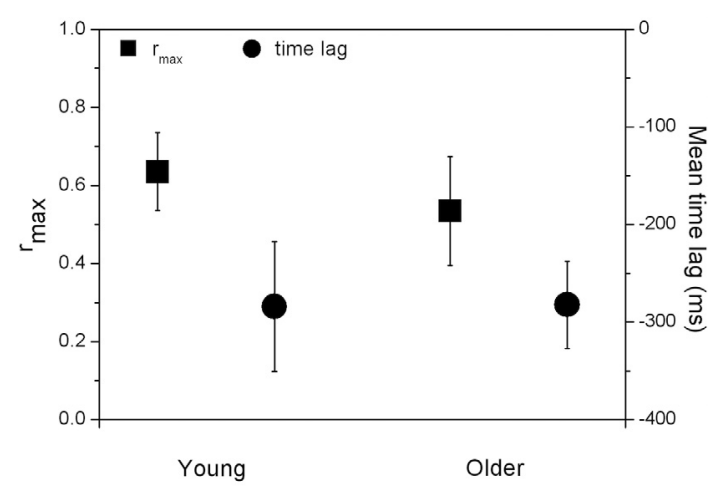

Fig. 4. Mean $( \pm S D)$ of the maximum cross-correlation coefficient $\left(r_{\max }\right)$ and time lag values of young and older adults during the light touch condition.

$\mathrm{p}=0.005$, partial $\eta^{2}=0.333$ ) directions, as older adults applied more force in all dimensions than young adults. On the other hand, no differences were identified between the groups for the fingertip tactile sensitivity by using Semmes-Weinstein monofilaments $(\mathrm{U}=33, \mathrm{p}>0.05)$.

Fig. 4 shows mean values of maximum cross-correlation coefficient $\left(\mathrm{r}_{\max }\right)$ and time lag for young and older adults during the light touch fingertip contact conditions. MANOVA revealed no group differences (Wilks' Lambda $=0.84, \mathrm{~F}_{2,19}=1.76$, $\mathrm{p}=0.199$, partial $\eta^{2}=0.156$ ) for the $r_{\max }$ and time-lag. On average, $r_{\max }$ and time lag were approximately 0.6 and -300 ms, respectively, indicating that the fingertip CP motion preceded whole body CP motion.

\subsection{Ankle passive motion and postural control}

Finally, there was a significant relationship between ankle passive motion sensitivity and postural control, collapsed data from both groups, in all experimental conditions (Table 1). Fig. 5 depicts scatter plots showing the relationship between postural control and ankle passive joint motion for both young and older adults. In general, larger CP sway was associated with larger ankle angular displacement, which was required to sense the motion and stop the passive motion apparatus; data from young adults were clustered in the left-lower corner, while data from older adults were clustered in the right-upper corner.

Although the MANCOVA still revealed a group effect (Wilks' Lambda $=0.436, \mathrm{~F}_{2,18}=11.63, \mathrm{p}<0.005$, partial $\eta^{2}=0.564$ ); and covariate (Wilks' Lambda $=0.703, \mathrm{~F}_{2,18}=3.80, \mathrm{p}=0.04$, partial $\eta^{2}=0.297$ ), univariate analyses demonstrated a group difference for MSA-ML $\left(\mathrm{F}_{1,19}=13.32, \mathrm{p}<0.005\right.$, partial $\eta^{2}=0.412$; covariate, $\mathrm{F}_{1,19}=5.05, \mathrm{p}=0.037$, partial $\left.\eta^{2}=0.014\right)$, but not for MSAAP $\left(F_{1,19}=0.23, p=0.637\right.$, partial $\eta^{2}=0.012$; covariate, $F_{1,19}=0.27, p=0.607$, partial $\left.\eta^{2}=0.210\right)$. These results indicated that ankle joint displacements accounted for the observed differences between groups in postural control in the AP direction.

\section{Discussion}

The main goal of the present study was to examine whether possibly less accurate detection of passive motion of ankle joint would account for larger sway in older adults. In addition, this study also examined the use of light touch by older adults to determine if it might be sufficient to overcome potentially less accurate cues coming from the lower limb. Our results revealed that although postural control was altered in older adults, they took advantage of fingertip light touch to reduce postural sway. The use of such sensory cue did not differ from young adults. Older adults also demonstrated a deteriorated sensitivity of lower limb passive motion, which was partially related to larger CP sway in both older and young adults.

Larger magnitude of body sway in older adults than in younger adults is a common finding (Blaszczyk et al., 1994; Collins et al., 1995; Prioli et al., 2005). It is particularly more evident in more demanding postural tasks (Prioli et al., 2006). Altered postural control performance in older adults is supposedly associated with peripheral sensory degradation due to natural aging processes

Table 1

Pearson correlation (r) and $p$-value between mean sway amplitude (MSA) in the anterior-posterior and medial-lateral directions and passive motion sensitivity of ankle joint during no touch and light touch conditions.

\begin{tabular}{|c|c|c|c|c|}
\hline \multirow[t]{3}{*}{ MSA } & \multicolumn{4}{|c|}{ Condition } \\
\hline & \multicolumn{2}{|c|}{ No touch } & \multicolumn{2}{|c|}{ Light touch } \\
\hline & $\mathrm{r}$ & $p$-value & $\mathrm{r}$ & $p$-value \\
\hline Anterior-posterior & 0.58 & 0.005 & 0.54 & 0.010 \\
\hline Medial-lateral & 0.48 & 0.024 & 0.51 & 0.016 \\
\hline
\end{tabular}



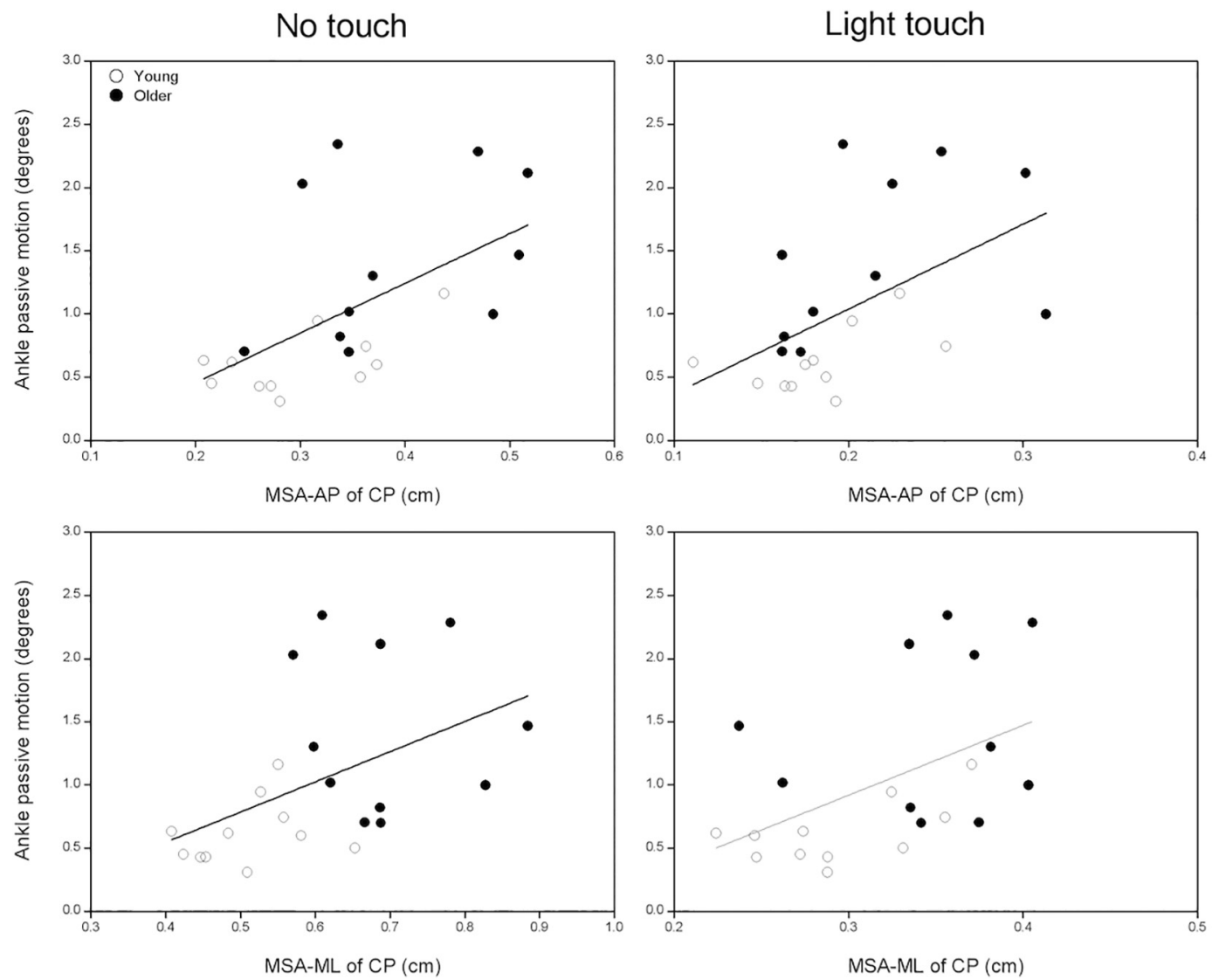

Fig. 5. Scatter plots between joint passive motion and mean sway amplitude (MSA) of the center of pressure (CP) in the anterior-posterior (upper panel) and medial-lateral (bottom panel) directions, during the no touch (left panel) and light touch (right panel) conditions, for both young (open circles) and older (filled circles) adults.

(Sturnieks et al., 2008). The findings of the present study advance our knowledge regarding the use of sensory information by older adults in several aspects.

First, we demonstrated that older adults take advantage and improve postural control performance when additional information is available. Specifically, older adults use fingertip sensory cues from a rigid surface to reduce postural sway (Baccini et al., 2007; Reginella et al., 1999; Tremblay et al., 2004). However, when we compare older adults to young adults in the light touch condition, they presented a similar magnitude of body sway, corroborating with our hypothesis that the enhancement of sensory cues from light touching a surface compensates for loss of peripheral proprioception from the lower limbs. The relationship established between the changes of position of CP and changes in the position of fingertip CP was similar for older and young adults (similar $r_{\text {max }}$ and time lags, Fig. 4). This means that older adults use changes in applied force to identify body sway changes and supposedly anticipate muscle activation as previously suggested for young adults (Jeka \& Lackner, 1995). A similar lag between changes in fingertip contact and body sway provides more evidence that indicates that older adults seem to preserve the capability of using sensory signals to produce successful behavior. Assuming the anticipatory strategy resulting from lightly touching a rigid surface observed in infants (Barela et al., 1999) and children (Bair, Barela, Whitall, Jeka, \& Clark, 2011), older adults were capable of using available sensory cues in a similar time scale as young adults. In contrast, recent evidence demonstrated that older adults suffer from a delayed proprioceptive afferent inflow to the cortex compared to young adults. Incoming cues from the periphery were weaker in older adults than those in young adults, when performing a task resembling the one used in the current study for passive movement detection (Toledo, Manzano, Barela, \& Kohn, 2016). These contradictory results could be attributed to task specificity and requirements, since the adaptation and use of cues from the fingertip did not require a quick, prompt response. Small time-scale differences, which might have occurred in the touch cues used in the current study, might not have been large enough to be shown.

Our results confirmed previous findings that demonstrated that older adults need larger ankle joint passive motion to detect it (Toledo \& Barela, 2010). These differences are related to the magnitude of upright stance sway, suggesting that older adults sway more and have poor postural control, which generates changes in body dynamics that are signaled to the CNS. Toledo et al. (2016) recently demonstrated the delayed inflow of proprioceptive cues to the cortex of older adults. Higher force level during fingertip contact of older adults, observed in the present and previous (Tremblay et al., 2004) studies, is another piece of evidence of peripheral sensory impairment. In the present study, older adults were capable of maintaining the force below the pre-defined level 
$(<1 \mathrm{~N})$, but they spontaneously adopted slightly higher values than that observed in young adults (Fig. 3). This suggested that their force detection threshold might be altered. A higher magnitude of vertical force exerted against the rigid surface could also be a sign of sensory deficits, compensating for the observed weaker proprioceptive signals arriving at the cortex (Toledo et al., 2016). Similarly, Cole and colleagues (1999) observed a higher magnitude of grip force (i.e., normal force component acting against the object surface) in older adults than in young adults when they performed a simple manipulation task (i.e., lifting and holding a grasped object). They related this change to the loss of fast adapting cutaneous mechanoreceptors (i.e., Meissner's corpuscles) and, consequently, to a deficit of sensitivity in the skin of the digits.

In addition to sample size, another limitation of the current study could be that, compared to the postural control task, passive motion of ankle joint was assessed in a different position and involved only one direction of motion. Nonetheless, our results indicated that changes in the peripheral sensory mechanisms providing less accurate signals to the CNS might account for the deficits in performance and behavior observed in the postural control of older adults. Larger passive motion of the ankle joints is related to a larger sway magnitude (correlation coefficients), which can account for group differences, particularly in the AP direction (direction that the passive motion was assessed), as shown by the MANCOVA results. This indicated that less accurate information coming from the ankle joint is one of the main contributors for such differences. More interestingly, this would explain, for instance, larger responses to visual manipulation in older adults than that in young adults (Jeka, Allison, \& Kiemel, 2010; Prioli et al., 2005; Wade, Lindquist, Taylor, \& Treat-Jacobson, 1995). Poor sensory cues from the lower limbs would be less informative. The CNS would be more influenced by visual manipulation, displaying larger sway due to the visual cue manipulation.

Considering that such sensory impairment might not be restricted to lower limbs (i.e., older adults applied more force with the fingertip on the touch bar), manipulating sensory cues from any channel would disrupt postural orientation to large extent in this population, as previously observed (Horak, Diener, \& Nashner, 1989; Woollacott, Shumway-Cook, \& Nashner, 1986). In addition, the positive effects of specific program intervention (Gauchard, Jeandel, Tessier, \& Perrin, 1999; Lord et al., 2005) and general physical activities (Gauchard, Lascombes, Kuhnast, \& Perrin, 2001; Lord, Menz, \& Tiedemann, 2003) on postural control performance may be due to improvement in the sensory cues that are provided by peripheral structures, which supplement the CNS with more accurate body dynamics information. Thus, conflicting sensory situations more appropriately (Prioli et al., 2005) are consequently solved by better adaptation to changing environmental conditions, as recently discussed (Anson \& Jeka, 2010).

\section{Conclusion}

Older adults can use additional sensory information coming from lightly contacting the fingertip with a rigid surface to improve postural control performance. The use of light touch may override peripheral sensory loss of the lower limbs, considering that older adults require larger motion around the ankle joints to detect changes in intersegmental configuration. Apart from being able to use light touch in an anticipatory manner, which is similar in young adults, older adults also present a similar magnitude of body sway as in young adults when light touch is available. This indicates that the enhancement of peripheral sensory cues due to fingertip contact to a stationary surface seems to contribute to improving postural control performance in this population. Overall, these results indicated that peripheral sensory deficits contribute to poor performance in postural control of older adults and that these deficits may be compensated by additional sensory cues, which provide enhanced information that is used in postural control.

\section{Acknowledgments}

This work was supported by the São Paulo Research Foundation - FAPESP (grant \#2009/15003-0).

\section{Appendix A. Supplementary data}

Supplementary data associated with this article can be found, in the online version, at http://dx.doi.org/10.1016/j.humov.2018. 06.001 .

\section{References}

Anson, E., \& Jeka, J. J. (2010). Sensory reweighting: A rehabilitative mechanism? In C. L. Armstrong, \& L. Morrow (Eds.). Handbook of medical neuropsychology (pp. 519-529). Amsterdam: Springer Science.

Baccini, M., Rinaldi, L. A., Federighi, G., Vannucchi, L., Paci, M., \& Masotti, G. (2007). Effectiveness of fingertip light contact in reducing postural sway in older people. Age and Ageing, 36(1), 30-35.

Bair, W. N., Barela, J. A., Whitall, J., Jeka, J. J., \& Clark, J. E. (2011). Children with developmental coordination disorder benefit from using vision in combination with touch information for quiet standing. Gait and Posture, 34(2), 183-190.

Barela, J. A., Jeka, J. J., \& Clark, E. J. (1999). The use of somatosensory information during the acquisition of independent upright stance. Infant Behavior and Development, 22, 87-102.

Barela, J. A., Jeka, J. J., \& Clark, J. E. (2003). Postural control in children: coupling to dynamic somatosensory information. Experimental Brain Research, 150(4), 434-442.

Blaszczyk, J., Lowe, D., \& Hansen, P. D. (1994). Ranges of postural stability and their changes in the eldery. Gait and Posture, $2,11-17$.

Bonfim, T. R., Grossi, D. B., Paccola, C. A., \& Barela, J. A. (2008). Additional sensory information reduces body sway of individuals with anterior cruciate ligament injury. Neuroscience Letters, 441(3), 257-260.

Cole, K. J., Rotella, D. L., \& Harper, J. G. (1999). Mechanisms for age-related changes of fingertip forces during precision gripping and lifting in adults. Journal of Neuroscience, 19(8), 3238-3247.

Collins, J. J., De Luca, C. J., Burrows, A., \& Lipsitz, L. A. (1995). Age-related changes in open-loop and closed-loop postural control mechanisms. Experimental Brain 
Research, 104(3), 480-492.

Cunha, B. P., Alouche, S. R., Araujo, I. M., \& Freitas, S. M. (2012). Individuals with post-stroke hemiparesis are able to use additional sensory information to reduce postural sway. Neuroscience Letters, 513(1), 6-11.

Dickstein, R., Shupert, C. L., \& Horak, F. B. (2001). Fingertip touch improves postural stability in patients with peripheral neuropathy. Gait and Posture, 14(3), $238-247$.

Gauchard, G. C., Jeandel, C., Tessier, A., \& Perrin, P. P. (1999). Beneficial effect of proprioceptive physical activities on balance control in elderly human subjects. Neuroscience Letters, 273(2), 81-84.

Gauchard, G. C., Lascombes, P., Kuhnast, M., \& Perrin, P. P. (2001). Influence of different types of progressive idiopathic scoliosis on static and dynamic postural control. Spine, 26(9), 1052-1058.

Holden, M., Ventura, J., \& Lackner, J. R. (1994). Stabilization of posture by precision contact of the index finger. Journal of Vestibular Research, 4(4), 285-301.

Horak, F. B., Diener, H. C., \& Nashner, L. M. (1989). Influence of central set on human postural responses. Journal of Neurophysiology, 62(4), 841-853.

Horak, F. B., \& Macpherson, J. M. (1996). Postural orientation and equilibrium. In L. B. Rowell, \& J. T. Shepard (Eds.). Handbook of physiology (pp. 255-292). New York: Oxford University Press.

Jeka, J. J., Allison, L. K., \& Kiemel, T. (2010). The dynamics of visual reweighting in healthy and fall-prone older adults. Journal of Motor Behavior, 42(4), 197-208.

Jeka, J. J., \& Lackner, J. R. (1994). Fingertip contact influences human postural control. Experimental Brain Research, 100(3), 495-502.

Jeka, J. J., \& Lackner, J. R. (1995). The role of haptic cues from rough and slippery surfaces in human postural control. Experimental Brain Research, 103(2), 267-276.

Jeka, J. J., Schoner, G., Dijkstra, T., Ribeiro, P., \& Lackner, J. R. (1997). Coupling of fingertip somatosensory information to head and body sway. Experimental Brain Research, 113(3), 475-483.

Lackner, J. R., DiZio, P., Jeka, J., Horak, F., Krebs, D., \& Rabin, E. (1999). Precision contact of the fingertip reduces postural sway of individuals with bilateral vestibular loss. Experimental Brain Research, 126(4), 459-466.

Lord, S. R., Clark, R. D., \& Webster, I. W. (1991). Postural stability and associated physiological factors in a population of aged persons. Journal of Gerontology, 46(2), M69-76.

Lord, S. R., \& Menz, H. B. (2000). Visual contributions to postural stability in older adults. Gerontology, 46, 306-310.

Lord, S. R., Menz, H. B., \& Tiedemann, A. (2003). A physiological profile approach to falls risk assessment and prevention. Physical Therapy, 83(3), 237-252.

Lord, S. R., Tiedemann, A., Chapman, k., Munro, B., Murray, S. M., \& Sherrington, C. (2005). The effects of an individualized fall prevention program on fall risk and falls in older people: A randomized, controlled trial. Journal of American Geriatrics Society, 53, 1296-1304.

Lord, S. R., \& Ward, J. A. (1994). Age-associated differences in sensori-motor function and balance in community dwelling women. Age and Ageing, 23, 452-460.

Maki, B. E., \& Mcllroy, W. E. (1996). Postural control in the older adult. Clinics in Geriatric Medicine, 12(4), 635-658.

Metcalfe, J. S., McDowell, K., Chang, T. Y., Chen, L. C., Jeka, J. J., \& Clark, J. E. (2005). Development of somatosensory-motor integration: an event-related analysis of infant posture in the first year of independent walking. Developmental Psychobiology, 46(1), 19-35.

Nashner, L. M. (1981). Analysis of stance posture in humans. In A. L. Towe, \& E. S. Luschei (Vol. Eds.), Motor coordination: handbook of behavioral neurology: Vol. 5, (pp. 527-565). New York, NY: Plenum Press.

Prioli, A. C., Cardozo, A. S., de Freitas Junior, P. B., \& Barela, J. A. (2006). Task demand effects on postural control in older adults. Human Movement Science, 25(3), 435-446.

Prioli, A. C., Freitas Junior, P. B., \& Barela, J. A. (2005). Physical activity and postural control in the elderly: Coupling between visual information and body sway. Gerontology, 51(3), 145-148.

Reginella, R. L., Redfern, M. S., \& Furman, J. M. (1999). Postural sway with earth-fixed and body-referenced finger contact in young and older adults. Journal of Vestibular Research, 9(2), 103-109.

Sturnieks, D. L., St George, R., \& Lord, S. R. (2008). Balance disorders in the elderly. Neurophysiologie Clinique, 38(6), 467-478.

Toledo, D. R., \& Barela, J. A. (2010). Sensory and motor differences between young and older adults: somatosensory contribution to postural control. Revista Brasileira de Fisioterapia, 14(3), 267-275.

Toledo, D. R., \& Barela, J. A. (2014). Age-related differences in postural control: Effects of the complexity of visual manipulation and sensorimotor contribution to postural performance. Experimental Brain Research, 232(2), 493-502.

Toledo, D. R., Manzano, G. M., Barela, J. A., \& Kohn, A. F. (2016). Cortical correlates of response time slowing in older adults: ERP and ERD/ERS analyses during passive ankle movement. Clinical Neurophysiology, 127(1), 655-663.

Tremblay, F., Mireault, A. C., Dessureault, L., Manning, H., \& Sveistrup, H. (2004). Postural stabilization from fingertip contact: I. Variations in sway attenuation, perceived stability and contact forces with aging. Experimental Brain Research [Experimentelle Hirnforschung. Expérimentation cérébrale]. 157(3), 275-285.

Wade, M. G., Lindquist, R., Taylor, J. R., \& Treat-Jacobson, D. (1995). Optical flow, spatial orientation, and the control of posture in the elderly. Journal of Gerontology, 50B(1), P51-P58.

Winter, D. A., Prince, F., Frank, J. S., Powell, C., \& Zabjek, K. F. (1996). Unified theory regarding A/P and M/L balance in quiet stance. Journal of Neurophysiology, 75(6), 2334-2343.

Woollacott, M., \& Shumway-Cook, A. (1990). Changes in posture control across the life span: A systems approach. Physical Therapy, $70(12), 799-807$.

Woollacott, M. H., Shumway-Cook, A., \& Nashner, L. M. (1986). Aging and posture control: Changes in sensory organization and muscular coordination. International Journal of Aging and Human Development, 23(2), 97-114. 